\title{
Improved Detection in CDMA for Biased Sources
}

\author{
Hadar Efraim, Nadav Yacov, Ori Shental, Ido Kanter* and David Saad
}

\begin{abstract}
We consider the detection of biased information sources in the ubiquitous Code-Division MultipleAccess (CDMA) scheme. We propose a simple modification to both the popular single-user matchedfilter detector and a recently introduced near-optimal message-passing based multiuser detector. This modification allows for detecting modulated biased sources directly, with no need for source coding. Analytical results and simulations with excellent agreement are provided, demonstrating substantial improvement in bit error rate in comparison with the unmodified detectors and the alternative of source compression. The robustness of error-performance improvement is shown under practical model settings, including bias estimation mismatch and finite-length spreading codes.
\end{abstract}

\section{Index Terms}

CDMA, biased sources, multiuser detection, source coding, bias mismatch.

* Corresponding Author. The first three authors contributed equally to this work.

H. Efraim, N. Yacov and I. Kanter are with the Minerva Center and Department of Physics, Bar-Ilan University, Ramat-Gan 52900, Israel (e-mail: \{hadaref,nadav.yacov,kanter\}@mail.biu.ac.il).

O. Shental is with the Center for Magnetic Recording Research (CMRR), University of California, San Diego (UCSD), 9500 Gilman Drive, La Jolla, CA 92093, USA (e-mail: oshental@ucsd.edu).

D. Saad is with the Neural Computing Research Group, Aston University, Birmingham B4 7ET, UK (e-mail: d.saad@aston.ac.uk).

Submitted To JPA. Revised June 9, 2007. 


\section{INTRODUCTION}

Direct-sequence spread-spectrum Code-Division Multiple-Access (CDMA) is used extensively in modern wireless communication systems and serves preeminently in commercial cellular networks. Typically in the uncoded CDMA literature [1], the binary information source, modulated for transmission over the channel, is assumed to be taken from an unbiased identically and independently distributed (i.i.d.) random process.

In practice, however, a substantial level of redundancy can often be observed in real-life uncoded sources (e.g., uncompressed binary images) which can be viewed as a bias in the generating Bernoulli distribution. In such cases, it would be reasonable to use binary source encoding. A source encoder is said to be optimal if it can eliminate all source redundancies and generate unbiased outputs. However, most existing practical source encoders, which are typically fixed-length encoders, are sub-optimal. Thus, the source encoder output still contains a certain level of bias, which can be further exploited in the transceiver design.

As for coded systems, it has been shown [2] that the empirical distribution of any 'good' error-correcting code ${ }^{1}$ converges to the channel's capacity-achieving input distribution. Hence, well-coded information sources, in the common case of binary-input additive white Gaussian noise (BI-AWGN) CDMA channel, must be unbiased ${ }^{2}$. However, bias can be found in practical coded CDMA systems in which not so 'good' codes are being employed, e.g., systematic Turbo codes [4]-[6], for which the systematic component of the code entails bias.

Therefore, tuning CDMA detection for biased sources is of major importance in both coded and uncoded systems. In this paper, we examine the commonly-used random spreading scheme, which lends itself to analysis and well describes CDMA with long signature sequences. We suggest a different approach for handling CDMA with biased sources than source compression.

We propose a scheme in which the source bias $m$ is assumed to be estimated by the receiver and is used for modifying, for instance, both naive single-user matched-filter (SUMF) and stateof-the-art multiuser CDMA detectors. This scheme can be categorized as a joint source codingmodulation approach, where the CDMA demodulation is performed utilizing side information

\footnotetext{
${ }^{1} \mathrm{~A}$ 'good' code is a code approaching capacity with asymptotically vanishing probability of error.

${ }^{2}$ As the capacity-achieving input distribution of the BI-AWGN channel is Bernoulli 1/2 [3].
} 
(i.e., bias) about the source. Based on large-system limit ${ }^{3}$ analysis, we find that a tuning, or correction, factor of the form $c \cdot \tanh ^{-1} m$ improves the detection of the biased source. For the SUMF (particularly suitable for the downlink) we find ${ }^{4} c \triangleq\left(\beta+\sigma^{2}\right)$, and for the tractable beliefpropagation based multiuser detector (particularly relevant for the uplink), recently introduced by Kabashima [7], we find $c \triangleq 1$. The latter is up-to-date the best known error performing tractable detector for random spreading CDMA in cases where the noise level is known, and has recently been extended to the general case where the noise level is unknown [8]. The derived scheme is shown to yield an improved error performance under practical system settings as finite-length spreading codes and bias estimation mismatch. The proposed scheme outperforms the alternative of applying source coding throughout a wide range of practical bias values. Furthermore, this improved detection scheme can be also used in processing biased feedback information in iterative multiuser decoding. No other detection scheme for biased sources has been proposed previously in the literature for CDMA.

\section{CDMA With Biased Sources}

Consider a $K$-user synchronous direct-sequence binary phase shift-keying (DS/BPSK) CDMA system employing random binary spreading codes of $N$ chips over an AWGN channel. The received signal sample of such a system can be described by

$$
y_{\mu}=\frac{1}{\sqrt{N}} \sum_{k=1}^{K} s_{\mu k} b_{k}+n_{\mu},
$$

where $s_{\mu k}= \pm 1(\mu=1, \ldots, N, k=1, \ldots, K)$ are the binary spreading chips being independently and equiprobably chosen. The deterministic chip waveform is assumed to be of unit energy; $b_{k}$ is the (possibly coded) information source binary symbol transmitted by the $k$ 'th user and is taken from a Bernoulli process with a non-zero bias of mean $-1 \leq m_{k} \leq 1$; $n_{\mu}$ is an AWGN sample taken from the Gaussian distribution $\mathcal{N}\left(0, \sigma^{2}\right)$. Hereinafter, the user's source bias $m_{k}$ is assumed to be (continuously re-) estimated at the receiver from the received signal (e.g., by using the likelihood of a Bernoulli process). Alternatively, the bias value can be reported periodically to the receiver via an auxiliary low-rate channel. The assumption of

\footnotetext{
${ }^{3}$ The limit where the number of users and processing gain tend to infinity but with a fixed ratio, which is defined as the system load $\beta$.

${ }^{4}$ Where $\beta$ and $\sigma^{2}$ are the system load and noise variance, respectively, as defined in the following Section II.
} 
(possibly erroneous) side information about the bias is particularly suitable for sources generated from known quasi-static distribution. We also assume a perfect power-control mechanism yielding unit energy transmissions. In the following, improvement of CDMA detection for biased sources is performed based on an asymptotic analysis, in which we assume that $N$ and $K$ are large, yet the system load factor $\beta \triangleq K / N$ is kept finite. Results for finite number of users, $K$, and practical spreading factor, $N$, under both perfect and erroneous bias estimation are presented in Section V. These results substantiate the validity and robustness of the suggested BER improvement to realistic systems.

\section{IMPROVING SINGLE-USER DETECTION}

Applying a SUMF for the CDMA detection problem results in binary decisions

$$
\hat{b}_{k}=\operatorname{sign}\left(\frac{1}{\sqrt{N}} \sum_{\mu=1}^{N} y_{\mu} s_{\mu k}\right)
$$

where $\operatorname{sign}(\cdot)$ is the hard-decision signum function. In order to adapt the SUMF for biased sources and improve its error performance, we reformulate the SUMF mechanism (2) to have the new form

$$
\hat{b}_{k}=\operatorname{sign}(\psi)
$$

with

$$
\psi \triangleq \frac{1}{\sqrt{N}} \sum_{\mu=1}^{N} y_{\mu} s_{\mu k}+\xi_{k} .
$$

For each user $k$, this decision rule of the SUMF is identical to its original form (2), up to an additive correction factor $\xi_{k}$, which is a function of the bias $m_{k}$ and the summation of the load $\beta$ and the noise variance $\sigma^{2}$. As we shall see, using an appropriate value of $\xi_{k}$, this minor change in the architecture of the SUMF yields a substantial enhancement in this detector's error performance for biased sources.

\section{A. Calculating $\xi_{k}$}

The SUMF output (before the hard-decision operation) can be rewritten as [1]

$$
\frac{1}{\sqrt{N}} \sum_{\mu} y_{\mu} s_{\mu k}=b_{k}+\frac{1}{N} \sum_{\mu} \sum_{k^{\prime} \neq k} s_{\mu k^{\prime}} s_{\mu k} b_{k^{\prime}}+\frac{1}{\sqrt{N}} \sum_{\mu} n_{\mu} s_{\mu k} .
$$


The last two terms in the SUMF output (5) are the multiple-access interference and the additive (white Gaussian) noise, respectively. In the large system analysis [1] according to the central limit theorem [9], these two random terms are distributed with $\mathcal{N}(0, \beta)$ and $\mathcal{N}\left(0, \sigma^{2}\right)$, respectively, thus their detrimental sum is taken from $\mathcal{N}\left(0, \beta+\sigma^{2}\right)$. Hence, for the new decision rule (3) the $k^{\prime}$ th user average probability for bit error, $P_{b_{k}}^{\mathrm{MF}}\left(\xi_{k}\right)$, is easily obtained as

$$
\begin{aligned}
P_{b_{k}}^{\mathrm{MF}}\left(\xi_{k}\right) & =\operatorname{Pr}\left(b_{k}=1\right) \operatorname{Pr}(\psi<0)+\operatorname{Pr}\left(b_{k}=-1\right) \operatorname{Pr}(\psi \geq 0) \\
& =\frac{1+m_{k}}{2} \mathcal{Q}\left(\frac{\xi_{k}+1}{\sqrt{\beta+\sigma^{2}}}\right)+\frac{1-m_{k}}{2}\left(1-\mathcal{Q}\left(\frac{\xi_{k}-1}{\sqrt{\beta+\sigma^{2}}}\right)\right),
\end{aligned}
$$

where the $Q$-function $\mathcal{Q}(\alpha) \triangleq \int_{\alpha}^{\infty} \exp \left(-x^{2} / 2\right) d x / \sqrt{2 \pi}$ is the complementary cumulative distribution function. The correction for optimal error performance under this scheme, $\xi_{k}^{\text {opt }}$, is obtained by finding the root of the derivative of $P_{b_{k}}^{\mathrm{MF}}\left(\xi_{k}\right)(6)$ w.r.t. $\xi_{k}$, resulting in

$$
\xi_{k}^{\mathrm{opt}}=\left(\beta+\sigma^{2}\right) \tanh ^{-1} m_{k}
$$

\section{Improving Multiuser Detection}

Recently, Kabashima [7] has introduced a tractable iterative CDMA multiuser detector which is based on the celebrated belief propagation algorithm (BP, [10], [11]). This novel algorithm exhibits considerably faster convergence than conventional multistage detection [12] without increasing computational cost significantly. It is considered to provide a nearly-optimal detection when the spreading factor $N$ is large and the noise level is known. Similarly to multistage detection, at each iteration cycle $t$ this detector computes tentative soft decisions $\eta_{k}^{t}$ for each user transmission, of the form

$$
\eta_{k}^{t}=\tanh \left(h_{k}^{t}\right)
$$

The parameters $\eta_{k}^{t}$ and $h_{k}^{t}$ ( $h_{k}^{0}$ is initialized by equating it to the SUMF output (5)) are coupled 
and being iteratively computed using the following recipe ${ }^{5}$

$$
\begin{aligned}
U_{k}^{t} & =A^{t} \sum_{l=1}^{K} W_{k l} \eta_{l}^{t}+A^{t} \beta\left(1-Q^{t}\right) U_{k}^{t-1}, \\
h_{k}^{t+1} & =R^{t} h_{k}^{0}-U_{k}^{t}+A^{t} \eta_{k}^{t}\left(1-Q^{t}\right) U_{\mu}^{t-1}, \\
R^{t} & =A^{t}+A^{t} \beta\left(1-Q^{t}\right) R^{t-1},
\end{aligned}
$$

where $W_{k l} \triangleq \sum_{\mu=1}^{N} s_{\mu k} s_{\mu l} / N, Q^{t} \triangleq \sum_{k=1}^{K}\left(\eta_{k}^{t}\right)^{2} / K, A^{t} \triangleq\left(\sigma^{2}+\beta\left(1-Q^{t}\right)\right)^{-1}$ and tentative hard-decisions are taken by $\hat{b}_{k}^{t}=\operatorname{sign}\left(\eta_{k}^{t}\right)$. Producing $\hat{b}_{k}^{t} \equiv \hat{b}_{k}^{t+1}, \forall k$, serves as the convergence criterion.

\section{A. Calculating $\xi_{k}$}

As in the previous section, a correction factor $\xi_{k}$ for bias-based error-performance improvement is incorporated within the detection algorithm. The marginalized likelihood and posterior at the t'th update are defined as [7]

$$
\begin{aligned}
P^{t}\left(y_{\mu} \mid b_{k},\left\{y_{\nu \neq \mu}\right\}\right) & \propto \frac{1+\hat{\eta}_{\mu k}^{t} b_{k}}{2}, \\
P^{t}\left(b_{k} \mid \mathbf{y}\right) & \propto \frac{1+\eta_{k}^{t} b_{k}}{2},
\end{aligned}
$$

respectively, where the vector $\mathbf{y}$ denotes the set of all $N$ observations, and $\eta_{\mu k}^{t}, \hat{\eta}_{\mu k}^{t}$ are per user (defined) internal parameters. Thus, in the biased case according to Bayes' law

$$
\frac{1+\eta_{k}^{t} b_{k}}{2}=P^{t}\left(b_{k} \mid \mathbf{y}\right)=\alpha_{k}\left(\prod_{\mu=1}^{N} P^{t}\left(y_{\mu} \mid b_{k},\left\{y_{\nu \neq \mu}\right\}\right)\right) \frac{1+m_{k} b_{k}}{2}
$$

where $\alpha_{k}$ is a normalization constant and the last term in the product is the $k$ 'th user source bit prior, which corresponds to the message bias.

Using $\tanh ^{-1}(x)=1 / 2 \ln ((1+x) /(1-x)), \eta_{k}^{t}$ can be extracted from expression (14) as $\eta_{k}^{t}=\tanh \left(\frac{1}{2} \ln \frac{1+\eta_{k}^{t}}{1-\eta_{k}^{t}}\right)=\tanh \left(\frac{1}{2} \ln \frac{\left(1+m_{k}\right) \prod_{\mu=1}^{N}\left(1+\hat{\eta}_{\mu k}^{t}\right)}{\left(1-m_{k}\right) \prod_{\mu=1}^{N}\left(1-\hat{\eta}_{\mu k}^{t}\right)}\right)=\tanh \left(\sum_{\mu=1}^{N} \tanh ^{-1} \hat{\eta}_{\mu k}^{t}+\tanh ^{-1} m_{k}\right)$.

\footnotetext{
${ }^{5}$ As we shall prove, finding the correction factor $\xi_{k}$ for this multiuser detector results only in modifying the update rule (8).
} Moreover, the devised bias correction $\xi_{k}$ is optimal for any BP-based detector and can be derived directly by adding the correct prior in the Bayesian scheme. The details of Kabashima's detector are introduced for completeness, in addition to the fact that this recipe is being implemented in full in the following Section V. 
Comparing this result to the above noted expression for $\eta_{k}^{t}(8)$, the new bias-based improvement version of relation (8) is now easily inferred

$$
\eta_{k}^{t}=\tanh \left(h_{k}^{t}+\xi_{k}\right)
$$

with $\xi_{k}=\tanh ^{-1} m_{k}$ regardless of the iteration index $t$. Unlike in the SUMF case, $\xi_{k}$ here is independent of $\beta$ and $\sigma^{2}$.

\section{RESUlTS AND Discussion}

In this section, the proposed scheme for biased sources is examined for the SUMF and Kabashima's multiuser detectors. Analytical and experimental results are obtained under both theoretical (large spreading factor and exact bias knowledge) and practical (finite $N$ and approximate bias estimation) model settings. Unless otherwise is specifically stated, all SUMF (Kabashima) detector results are obtained for $\operatorname{load} \beta=0.8(0.5)$ and signal-to-noise ratio, $\mathrm{SNR} \triangleq 10 \log _{10}\left(1 / \sigma^{2}\right)=2 \mathrm{~dB}(6 \mathrm{~dB})$, while simulation results are averaged over sufficiently large ensemble of 4000 (2000) computer-simulated randomly-spread AWGN CDMA systems with long spreading factor $N=1000$ (2000). The standard deviation of all simulation results was small, thus omitted from the figures. From this point and on, sources with identical bias are assumed, thus omitting subscript $k$ from $m, \xi, P_{b}(\xi)$ and so on.

Fig. 1-(a) displays the normalized bit error rate (BER) (6) for the SUMF (3)-(4) detector as a function of the $\operatorname{bias}^{6} \mathrm{~m}$. As we are less interested in the well-known absolute error performance [1], [7] of the examined detectors, the BER is normalized by the probability of error if no bias-based modification (2) is applied (i.e., the unmodified detectors without bias side information). Thus, Fig. 1-(a) depicts the analytical improvement in performance (for large $N)$ due to the proposed scheme. It also presents empirical BER results in two different bias estimation settings. First, a perfect side information on the bias is assumed at the receiver. These experimental results agree perfectly with the analytical BER curve. Also drawn is the simulated normalized BER under the realistic model of mismatch in bias estimation. Here, a mismatch of $+20 \%$ and $-20 \%$ is examined, i.e., the detector assumes a bias which is $20 \%$ higher/lower than its true value. Clearly, the proposed scheme still suggests a substantial improvement in BER in this practical setting.

\footnotetext{
${ }^{6}$ Due to symmetry, only non-negative bias is shown in the figures.
} 


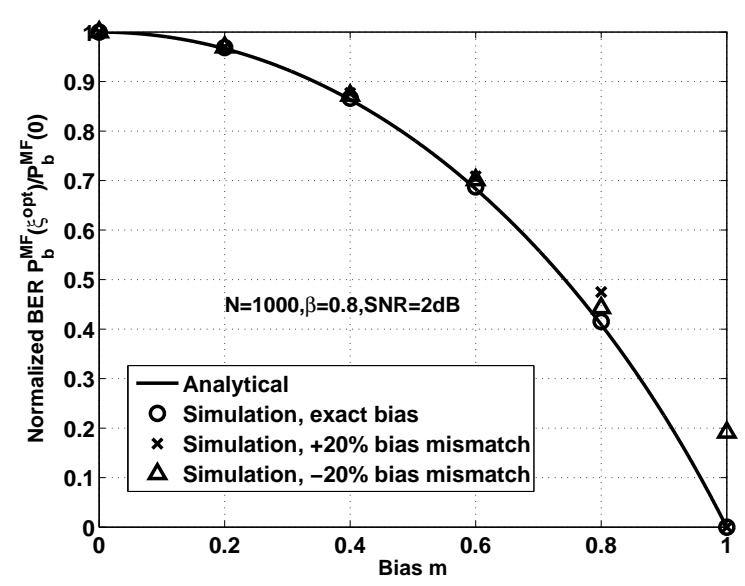

(a)

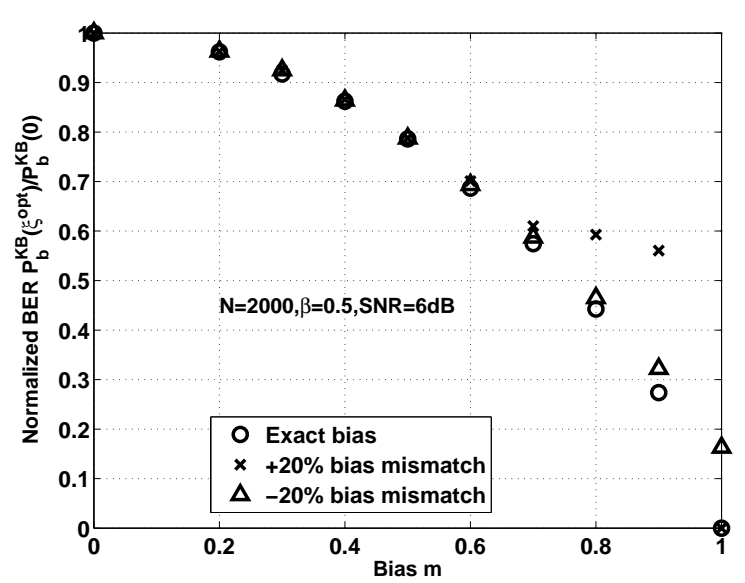

(b)

Fig. 1. (a) Normalized BER, $P_{b}^{\mathrm{MF}}\left(\xi^{o p t}\right) / P_{b}^{\mathrm{MF}}(0)$, for the SUMF detector as a function of the bias $m$ for $N=1000, \beta=0.8$ and $\mathrm{SNR}=2 \mathrm{~dB}$. Analytical BER (solid line), computed according to expression (6), is compared to simulated BER (circles) assuming the exact source bias is known to the receiver. Also drawn ( $\times$ and $\Delta$-marks) are the simulated BER curves under bias mismatch of $\pm 20 \%$. Simulation results are averaged over 4000 systems. (b) Simulated normalized $\mathrm{BER}, P_{b}^{\mathrm{KB}}\left(\xi^{o p t}\right) / P_{b}^{\mathrm{KB}}(0)$, for Kabashima's (KB) near-optimal detector [7] as a function of the bias $m$ for $N=2000, \beta=0.5$, SNR $=6 \mathrm{~dB}$ and averaged over 2000 systems. Simulated BER assuming exact bias (circles) knowledge at the receiver is compared to BER achieved under bias mismatch of $\pm 20 \%$ ( $\times$ and $\Delta$-marks).

Evidently, the normalized BER decreases down to zero for $m=1$, as knowing only the bias in this case trivially entails the transmitted binary symbols (all ' 1 's). Note that even for small bias values there is still some room for BER improvement due to the proposed scheme.

Simulation ${ }^{7}$ results for the improvement due to the proposed modification (16) of Kabashima's (KB) multiuser detector (9)-(11) are drawn in Fig. 1-(b) under exact and approximate bias knowledge settings. Both mismatch curves exhibit BER improvement monotonically increasing with the bias value, with or without exact bias estimation at the receiver. Overestimation is empirically observed as relatively more detrimental than underestimation for large bias values and these two mismatch cases are not symmetric. Still both mismatch cases demonstrate a significant improvement in BER.

Fig. 2-(a) presents the normalized simulated BER as a function of bias mismatch $(m=0.2,0.8)$ for both the SUMF and Kabashima's multiuser detectors. The robustness of the proposed scheme

\footnotetext{
${ }^{7}$ To this date, there is no exact asymptotic error performance analysis published for this detector.
} 


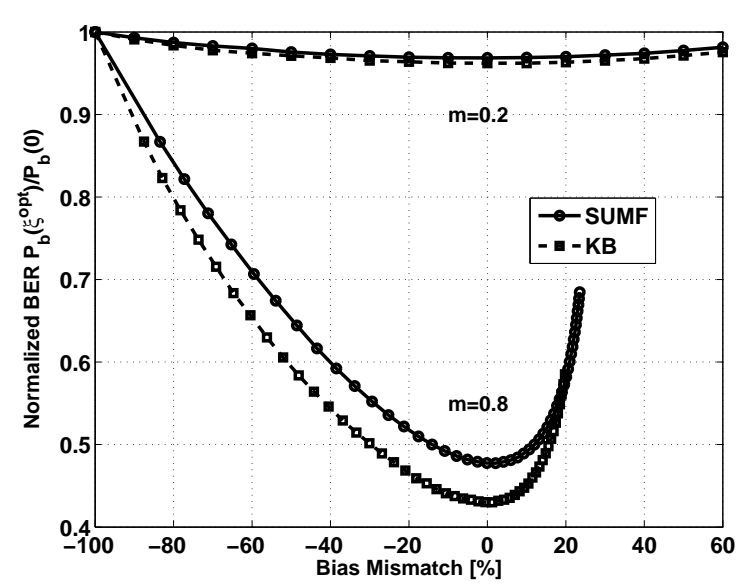

(a)

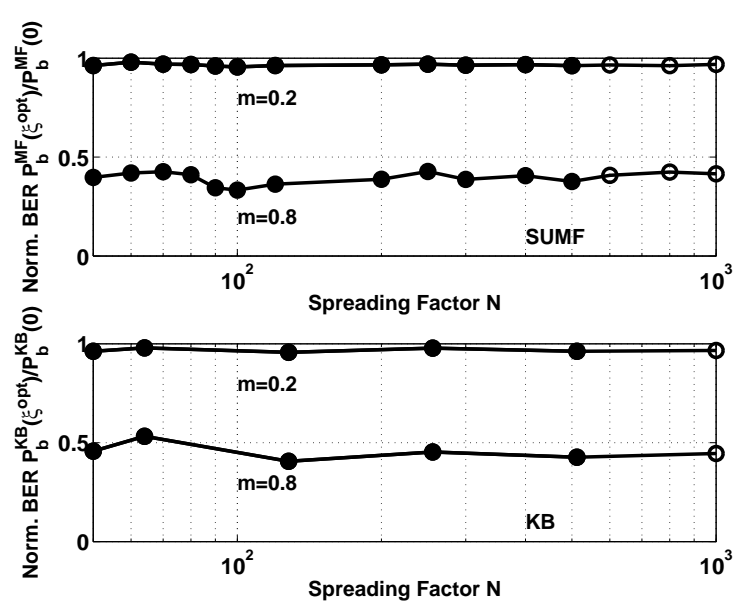

(b)

Fig. 2. (a) Simulated normalized BER, $P_{b}\left(\xi^{o p t}\right) / P_{b}(0)$, for the SUMF (circles and solid line) and Kabashima's multiuser (squares and dashed line) detectors as a function of the bias mismatch (in \%) for both $m=0.2$ (upper curves) and $m=0.8$ (lower curves). Averaged over $10^{4}$ simulated systems. (b) Normalized BER for SUMF (upper curves) and Kabashima's (lower curves) detectors as a function of the spreading factor $N$ for both $m=0.2$ and $m=0.8$ (10 ${ }^{4}$ simulated systems). Full circles denote practical $N$ values. In both figures, the lines are guides to the eye.

is observed over a wide range of bias mismatch, yielding BER improvement even under strong bias estimation error.

Up to this point, we have investigated the proposed scheme under the theoretical assumption of infinite (or at least very long) spreading codes. In order to verify its feasibility to practical CDMA settings, the BER performance under finite-length spreading codes (i.e., $N \leq 512$ ) has been examined. The upper curves in Fig. 2-(b) display the simulated normalized BER for SUMF as a function of the spreading factor $N$ for both relatively low bias, $m=0.2$, and high bias, $m=0.8$ ( $10^{4}$ simulated systems). The improvement in detection due to the proposed scheme for practical values of $N$ (full circles) is found to be (qualitatively) the same as the one obtained for large spreading factor (e.g., $N=1000$, empty circle). Similar results are obtained for Kabashima's multiuser detector (lower curves). Thus, the proposed scheme remains attractive for realistic finite spreading gains.

For Kabashima's iterative detector, we have evaluated not only the improvement in error performance, but also the reduction in the total number of iterations, $\tau\left(\xi^{\text {opt }}\right)$, required for convergence (which is an important factor in the implementation of such algorithms) induced 


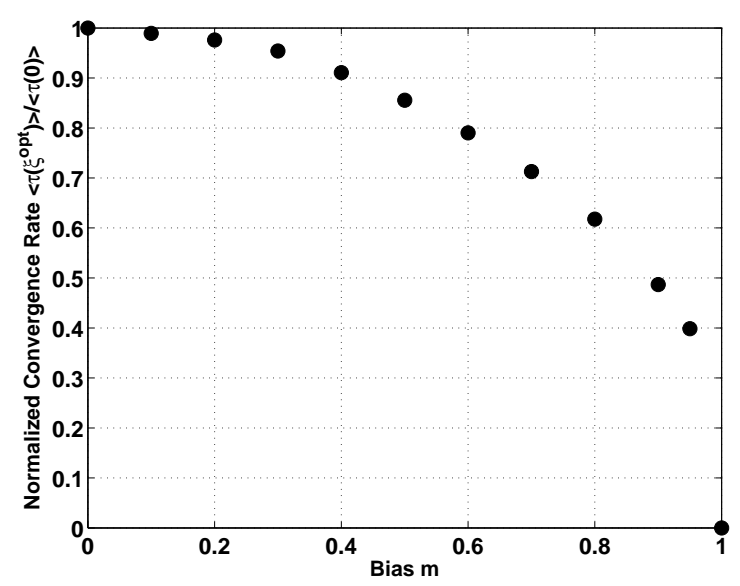

(a)

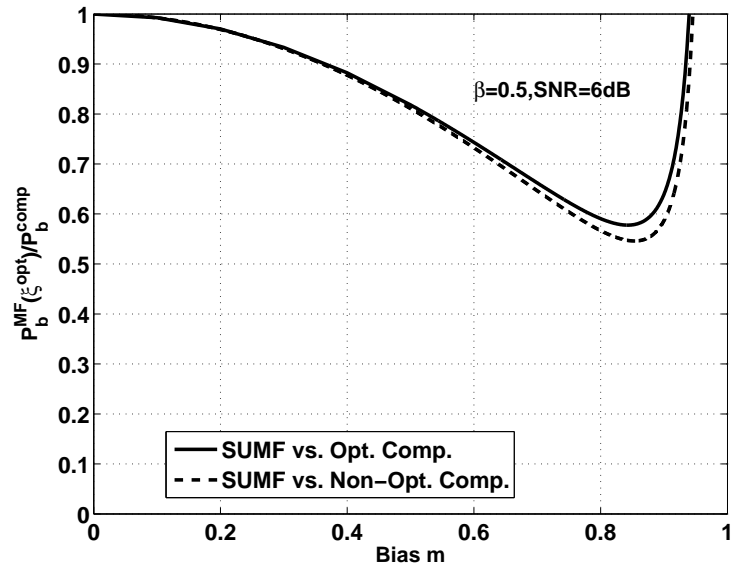

(b)

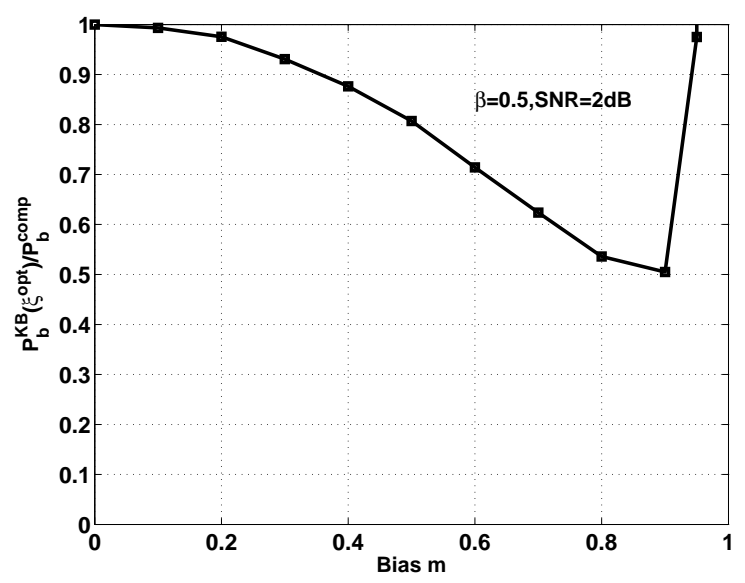

(c)

Fig. 3. (a) Normalized average convergence rate, $\left\langle\tau\left(\xi^{o p t}\right)\right\rangle /\langle\tau(0)\rangle$, as a function of the bias $m$ (for 1500 simulated systems). (b) SUMF BER comparison for the proposed scheme versus optimal and non-optimal (i.e., 5\% above the entropy) compression as a function of bias $m(\beta=0.5$ and SNR $=6 \mathrm{~dB})$. (c) Simulated BER comparison of Kabashima's detector for the proposed modification versus optimal compression as a function of bias $m(\beta=0.5$ and $\mathrm{SNR}=2 \mathrm{~dB})$. The squares denote simulation results, while the lines are only guides to the eye.

by the proposed mechanism. This improvement in the average convergence rate $\left\langle\tau\left(\xi^{\text {opt }}\right)\right\rangle(\langle\cdot\rangle$ denotes averaging operation), w.r.t. the number of iterations $\langle\tau(\xi=0)\rangle$ required in the standard case when equiprobable source information bits are assumed, is shown in Fig. 3-(a) (for 1500 simulated systems).

As previously stated, the mainstream alternative to our approach is source coding, or compression [13]. In order to evaluate the attractiveness of the proposed scheme, we compare its 
bit error probability $P_{b}^{\mathrm{MF}}\left(\xi^{\mathrm{opt}}, \sigma, \beta\right)$, when applied to a simple SUMF, to the bit error probability $P_{b}^{\text {comp }}\left(\xi=0, \sigma^{\text {comp }}, \beta^{\text {comp }}\right)$ achieved by (ordinary) SUMF detection of the transmission of unbiased (optimally) compressed source bits. As after optimal compression no bias-based improvement can be performed (i.e., $m=0$ ), the ratio between these two probabilities is given by

$$
\begin{aligned}
\frac{P_{b}^{\mathrm{MF}}}{P_{b}^{\text {comp }}} & =\frac{P_{b}^{\mathrm{MF}}\left(\xi^{\mathrm{opt}}, \sigma, \beta\right)}{P_{b}^{\mathrm{comp}}\left(\xi=0, \sigma^{\mathrm{comp}}=\sigma \sqrt{H_{b}(m)}, \beta^{\mathrm{comp}}=\beta H_{b}(m)\right)} \\
& =\frac{H_{b}(m) P_{b}^{\mathrm{MF}}\left(\xi^{\mathrm{opt}}, \sigma, \beta\right)}{P_{b}^{\mathrm{MF}}\left(\xi=0, \sigma^{\mathrm{comp}}=\sigma \sqrt{H_{b}(m)}, \beta^{\mathrm{comp}}=\beta H_{b}(m)\right)},
\end{aligned}
$$

where $H_{b}(m) \triangleq-0.5\left((1+m) \log _{2}((1+m) / 2)+(1-m) \log _{2}((1-m) / 2)\right)$ denotes the binary source's entropy. In computing the ratio (17), fair comparison dictates taking into consideration the differences in information rates and available transmission power between these two approaches, as follows. Note that as compression results in $H_{b}(m)$ times fewer compressed bits, they can be transmitted using an $H_{b}(m)$ times lower bandwidth and a $1 / \sqrt{H_{b}(m)}$ times higher transmission amplitude per bit. To eliminate this unfairness in the comparison, we have assumed that the compressed bits are conveyed under an effectively $\sqrt{H_{b}(m)}$ times lower noise level and $H_{b}(m)$ times lower load $\beta$. Also, in computing $P_{b}^{\text {comp }}$ when we assume an optimal source code, asymptotically speaking, a single error in detecting a compressed bit leads, on average, to $1 / H_{b}(m)$ errors in the uncompressed information.

Fig. 3-(b) presents the analytical BER ratio (17) as a function of the bias $m$ for $\beta=0.5$ and $\mathrm{SNR}=6 \mathrm{~dB}$. Interestingly, applying the proposed bias-based improvement to the SUMF is superior to the optimal compression alternative almost throughout the whole range of bias values. The performance gap is maximized for $m \sim 0.85$, giving over $40 \%$ BER improvement. Only for highly biased sources $(m \gtrsim 0.94$ ) compression is found to be advantageous. Similar comparison results (Fig. 3-(c)) are obtained when CDMA demodulation is performed using the nearly-optimal multiuser detector of Kabashima (for $\beta=0.5$ and $\mathrm{SNR}=2 \mathrm{~dB}$ ). In other words, the proposed joint source coding-modulation typically outperforms the separation into optimal compression and (SUMF/KB) demodulation.

Fig. 3-(b) also demonstrates the increasing superiority of the modified SUMF over non-optimal compression. A sub-optimal fixed-length source encoder compressing at $5 \%$ above entropy is assumed. Again, this curve is obtained using the analytical ratio (17), where the optimal 
compression rate $H_{b}(m)$ is now replaced by the sub-optimal rate $(1+0.05) H_{b}(m)$.

In this context, one should bear in mind that while standard compression exhibits a substantial delay and demands an excessive computational cost at both the transmitter and receiver, the proposed scheme does not significantly affect the transmitter. Moreover, the modification of existing detectors boils down to a simple correction within the detectors architecture. For the popular SUMF and the examined attractive multiuser detector [7] this correction is found to have the simple form $c \cdot \tanh ^{-1} m$.

In addition, the output bitstream of practical compression schemes, as opposed to optimal compression, still leaves some non-accounted and non-negligible bias, which can be further utilized by adopting our scheme. A prerequisite to the implementation of the described scheme is (not necessarily perfect) side information about the source bias being available to the receiver. Thus, the exact bias, or its estimate, can be computed (delivered) at (to) the receiver, incorporated into the detection process and render, as shown, a significant improvement in error performance.

\section{REFERENCES}

[1] S. Verdú, Multiuser Detection. Cambridge, UK: Cambridge University Press, 1998.

[2] S. Shamai (Shitz) and S. Verdú, “The empirical distribution of good codes," IEEE Trans. Inform. Theory, vol. 43, no. 3, pp. 836-846, May 1997.

[3] T. Tanaka, "A statistical-mechanics approach to large-system analysis of CDMA multiuser detectors," IEEE Trans. Inform. Theory, vol. 48, pp. 2888-2910, Nov. 2002.

[4] C. Berrou, A. Glavieux, and P. Thitimajshima, "Near Shannon limit error-correcting coding and decoding: Turbo codes," in in Proc. IEEE Int. Conf. Commun (ICC) (Geneva, Switzerland), Geneva, Switzerland, 1993, pp. 1064-1070.

[5] G. C. Zhu and F. Alajaji, "Turbo codes for non-uniform memoryless sources over noisy channels," IEEE Commun. Lett., vol. 6, no. 2, pp. 64-66, Feb. 2002.

[6] _ - "Design of Turbo codes for non-equiprobable memoryless sources," in Proc. 39th Allerton Conf. on Communications, Control and Computing, Monticello, IL, USA, Oct. 2001.

[7] Y. Kabashima, "A CDMA multiuser detection algorithm on the basis of belief propagation,” J. Phys. A: Math. Gen., vol. 36, pp. 11 111-11 121, Oct. 2003.

[8] J. P. Neirotti and D. Saad, "Improved message passing for inference in densely connected systems," Europhys. Lett., vol. 71, no. 5, pp. 866-872, Sept. 2005.

[9] R. S. Ellis, Entropy, Large Deviations, and Statistical Mechanics. New York: Springer-Verlag, 1985.

[10] J. Pearl, Probabilistic Reasoning in Intelligent Systems: Networks of Plausible Inference. San Francisco: Morgan Kaufmann, 1988.

[11] M. Opper and D. Saad, Advanced Mean Field Methods: Theory and Practice. Cambridge, MA: MIT Press, 2001.

[12] J. G. Andrews, "Interference cancellation for cellular systems: A contemporary overview," IEEE Wireless Commun. Mag., pp. 19-29, Apr. 2005. 
[13] T. M. Cover and J. A. Thomas, Elements of Information Theory. John Wiley and Sons, 1991. 